\title{
Implications of a light Higgs in composite models
}

\author{
Michele Redi ${ }^{a, b}$ and Andrea Tesi ${ }^{c}$ \\ ${ }^{a}$ CERN, Theory Division, \\ CH-1211, Geneva 23, Switzerland \\ ${ }^{b}$ INFN, Sezione di Firenze, \\ Via G. Sansone, 1; I-50019 Sesto Fiorentino, Italy \\ ${ }^{c}$ Scuola Normale Superiore, \\ Piazza dei Cavalieri, 7; I-56126 Pisa, Italy \\ E-mail: michele.redi@cern.ch, andrea.tesi@sns.it
}

ABSTRACT: We study the Higgs mass in composite Higgs models with partial compositeness, extending the results of ref. [1] to different representations of the composite sector for $\mathrm{SO}(5) / \mathrm{SO}(4)$ and to the coset $\mathrm{SO}(6) / \mathrm{SO}(5)$. For a given tuning we find in general a strong correlation between the mass of the top partners and the Higgs mass, akin to the one in supersymmetry. If the theory is natural a Higgs mass of $125 \mathrm{GeV}$ typically requires fermionic partners below $\mathrm{TeV}$ which might be within the reach of the present run of LHC. A discussion of CP properties of both cosets is also presented.

KeYwords: Higgs Physics, Beyond Standard Model, Technicolor and Composite Models

ARXIV EPRINT: 1205.0232 


\section{Contents}

1 Introduction 1

2 Coset space $\mathrm{SO}(5) / \mathrm{SO}(4) \quad 2$

2.1 Gauge lagrangian 3

$2.2 \mathrm{CHM}_{5}$

$\begin{array}{lll}2.2 .1 & \text { Composite right-handed quarks } & 6\end{array}$

$\begin{array}{lll}2.3 & \mathrm{CHM}_{10} & 7\end{array}$

3 Coset space $\mathrm{SO}(6) / \mathrm{SO}(5) \quad 9$

3.1 Gauge sector 9

$\begin{array}{lll}3.2 & \text { Fermionic sector } & 10\end{array}$

$\begin{array}{lll}3.3 & \text { Higgs and singlet mass } & 10\end{array}$

$\begin{array}{ll}3.4 \text { Modified couplings } & 12\end{array}$

4 Discussion 13

$\begin{array}{lr}\text { A Basic formulas } & \mathbf{1 5}\end{array}$

$\begin{array}{lll}\text { A.1 } & \mathrm{SO}(5) / \mathrm{SO}(4) & 15\end{array}$

$\begin{array}{lll}\text { A.1.1 } & \mathrm{CHM}_{5} & 16\end{array}$

$\begin{array}{lll}\text { A.1.2 } \mathrm{CHM}_{10} & 16\end{array}$

$\begin{array}{lll}\text { A.2 } & \mathrm{SO}(6) / \mathrm{SO}(5) & 17\end{array}$

$\begin{array}{ll}\text { B Potential } & 18\end{array}$

\section{Introduction}

We are likely at the dawn of the discovery of a light Higgs boson [2, 3]. A pressing question is whether the Higgs is Standard Model-like, or if there is new dynamics that stabilizes the electro-weak scale.

Two scenarios are at present the most compelling solutions of the hierarchy problem: supersymmetry and strong dynamics where the Higgs is a bound state of new strong interactions [4-8]. In the first at least new colored scalars, the stops, are expected below the $\mathrm{TeV}$ scale if the theory shall remain natural, while other supersymmetric partners could be heavier. If strong dynamics in the form of a Composite Higgs $(\mathrm{CH})$ boson is responsible for electro-weak symmetry breaking, we also expect new resonances around the $\mathrm{TeV}$ scale, with the same statistics as the SM fields in this case. But how heavy? This quantitative question is obviously crucial for the prospect of detecting the new states at the LHC. As we will see in a large class of models a conclusion similar to SUSY applies even quantitatively. Namely, if a light Higgs will be discovered, for a similar tuning as in 
SUSY new fermionic resonances must be present below the TeV scale to cut-off the top loop quadratic divergence. Vector resonances could instead be in the multi-TeV range, as hinted by precision tests, $S$ parameter in particular. In other words a hierarchical spectrum is predicted.

In this note we will focus on models where the Higgs is a pseudo-Goldstone boson as this is the only clear logic, beside supersymmetry, that allows one to obtain a naturally light scalar, see [12] for a review. Two patterns stand out phenomenologically, the minimal composite $\mathrm{SO}(5) / \mathrm{SO}(4)$ [8] delivering a single Higgs boson and $\mathrm{SO}(6) / \mathrm{SO}(5)$ that in addition produces a CP odd singlet [9]. Extended Higgs sectors are also possible [10] but we will not consider them here. Within this class we will consider scenarios with partial compositeness where the Higgs potential is generated by the couplings to the SM fields that explicitly break the global symmetries of the strong sector.

To address quantitatively the question of the mass we will use the simplified description of CH models with partial compositeness developed in [1] (see [11] for an alternative construction and [13-17] for related work). In this framework only the lightest resonances of the strong sector are introduced allowing a model independent analysis. This turns out to be sufficient to render the potential finite. In particular the quartic coupling of the Higgs that determines the Higgs mass is calculable in terms of masses and couplings of the new resonances. Other resonances could also be included with similar results for what concerns the Higgs mass. The results are comparable to the ones of 5D models for similar choices of parameters but other regions of parameter space are also explored. While not true in general, in all the models considered the top Yukawa controls the Higgs mass so that a relation between the fermionic partners and the tuning is obtained analogous to the one in supersymmetry for the stops.

The paper is organized as follows. In section 2 after briefly reviewing the simplified approach of ref. [1], we study the Higgs and fermionic partners mass in the minimal coset $\mathrm{SO}(5) / \mathrm{SO}(4)$ with fermions in the $\mathbf{5}$ and in the $\mathbf{1 0}$ representations. We also consider the possibility, motivated by flavor, that right-handed light quarks are strongly composite which generates new contributions to the potential but with similar results for the spectrum. Section 3 is devoted to the study of the coset space $\mathrm{SO}(6) / \mathrm{SO}(5)$. The mass of the Higgs is similar to the minimal $\mathrm{CH}$ while the singlet is typically heavier. We investigate in detail the $\mathrm{CP}$ properties of this model showing that the CP symmetry is not broken spontaneously nor explicitly by the coupling to the SM fermions. Conclusions and a discussion of the analogies with supersymmetry is in section 4 . The relevant formulas used in the paper are collected in appendix A. Some analytic formulas and intuition for the potential are in appendix B.

\section{Coset space $\mathrm{SO}(5) / \mathrm{SO}(4)$}

In ref. [1] we presented an effective lagrangian that encodes all the relevant features of CHM with partial compositeness and contains only the relevant degrees of freedom possibly accessible at the LHC (see also ref. [11]). Our simplified model contains for each SM fermion a single Dirac fermion in a representation of the global symmetry of the composite 
sector. As in the 5D models SM chiral fermions are associated to independent multiplets. Within this framework many observables of interest are calculable. In particular the Higgs potential, UV divergent in the low energy effective theory, is finite due to the presence of the resonances and can be used to estimate the Higgs mass up to model dependent effects. This reproduces results similar to 5D models for analogous choices of parameters.

The construction can be applied to any CHM. We start considering the simplest coset $\mathrm{SO}(5) / \mathrm{SO}(4)$ with the most popular choices of the embedding of fermions, the $\mathbf{5}$ and the 10. This pattern of symmetry breaking was studied in [1] and we refer to that paper for the technical details.

\subsection{Gauge lagrangian}

In the gauge sector the lagrangian reads [1],

$$
\begin{aligned}
\mathcal{L}_{\text {gauge }}= & -\frac{1}{4 g_{0}^{2}} F_{\mu \nu}^{a} F_{\mu \nu}^{a}-\frac{1}{4 g_{0 Y}^{2}} Y_{\mu \nu} Y^{\mu \nu} \\
& +\frac{f_{1}^{2}}{4} \operatorname{Tr}\left|D_{\mu} \Omega\right|^{2}+\frac{f_{2}^{2}}{2}\left(D_{\mu} \Phi\right)^{T}\left(D^{\mu} \Phi\right)-\frac{1}{4 g_{\rho}^{2}} \rho_{\mu \nu}^{A} \rho^{A \mu \nu}
\end{aligned}
$$

where $\Omega$ is an $\mathrm{SO}(5)$ matrix parametrizing the coset $\mathrm{SO}(5)_{L} \times \mathrm{SO}(5)_{R} / \mathrm{SO}(5)_{L+R}$, and $\Phi$ is a five dimensional unit vector whose VEV breaks spontaneously $\mathrm{SO}(5)$ to $\mathrm{SO}(4)$. The composite spin-1 resonances $\rho_{\mu}$ are introduced as gauge fields of the diagonal subgroup of $G_{R}+G$ and the SM fields gauge the electro-weak subgroup of the $\mathrm{SO}(5)_{L}$ global symmetry of the composite sector,

$$
D_{\mu} \Omega=\partial_{\mu} \Omega-i A_{\mu} \Omega+i \Omega \rho_{\mu}, \quad D_{\mu} \Phi=\partial_{\mu} \Phi-i \rho_{\mu} \Phi
$$

where $A_{\mu}$ are the elementary gauge fields. The physical decay constant of the 4 GBs (the Higgs) is,

$$
f^{2}=\frac{f_{1}^{2} f_{2}^{2}}{f_{1}^{2}+f_{2}^{2}}
$$

To reproduce the hyper-charge assignments of fermions a $\mathrm{U}(1)_{X}$ symmetry should also be included so that $Y=T_{R}^{3}+X$.

\section{$2.2 \mathrm{CHM}_{5}$}

Each SM quark is coupled to a distinct Dirac fermion in an $\mathrm{SO}(5)$ representation. The spontaneous breaking $\mathrm{SO}(5) / \mathrm{SO}(4)$ allows couplings between fermions associated the left and right chiralities of SM fields that will eventually generate SM Yukawas. For the model where the fermions are in the $\mathbf{5}$ of $\mathrm{SO}(5)\left(\mathrm{CHM}_{5}[29]\right)$ the lagrangian of the third generation reads,

$$
\begin{aligned}
\mathcal{L}^{\mathrm{CHM}_{5}}= & \bar{q}_{L}^{e l} i \not D^{e l} q_{L}^{e l}+\bar{t}_{R}^{e l} i \not D^{e l} t_{R}^{e l} \\
& +\Delta_{t_{L}} \bar{q}_{L}^{e l} \Omega \Psi_{T}+\Delta_{t_{R}} \bar{t}_{R}^{e l} \Omega \Psi_{\widetilde{T}}+\text { h.c. } \\
& +\bar{\Psi}_{T}\left(i \not D^{\rho}-m_{T}\right) \Psi_{T}+\bar{\Psi}_{\widetilde{T}}\left(i \not D^{\rho}-m_{\widetilde{T}}\right) \Psi_{\widetilde{T}} \\
& -Y_{T} \bar{\Psi}_{T, L} \Phi \Phi^{T} \Psi_{\widetilde{T}, R}-m_{Y_{T}} \bar{\Psi}_{T, L} \Psi_{\widetilde{T}, R}+\text { h.c. } \\
& +(T \rightarrow B) .
\end{aligned}
$$


The elementary quarks $q_{L}^{e l}$ and $t_{R}^{e l}$ couple to two different Dirac fermions $\Psi_{T}$ and $\Psi_{\widetilde{T}}$, in the 5 representation of $\mathrm{SO}(5)$, via mass mixing $\Delta_{t_{L}}$ and $\Delta_{t_{R}}$ that respect the SM gauge symmetry. The terms in the fourth line break spontaneously $\mathrm{SO}(5) / \mathrm{SO}(4)$ and contain interactions with the GBs. We retain the only terms with a certain chirality as necessary to generate the SM Yukawas (see [1] for more details). We recall that in $\mathrm{CHM}_{5}$ the SM quark doublet must couple to two composite fermions with different charge under $\mathrm{U}(1)_{X}$ to generate Yukawa of the top and bottom quark. The couplings of the down sector (to which we refer in (2.4) in the last line) however will not be important here. Within the anarchic scenarios [8] the Higgs potential is dominated by the third generation because the mixings of the light generations are small however this might not be true in general and we provide an example below.

In general $m_{Y_{T}}$ and $Y_{T}$ are complex parameters. One phase can be reabsorbed with a redefinition of the composite fields while the relative phase remains as a physical $\mathrm{CP}$ violating phase. This describes a strong sector that breaks CP. The same holds when the coupling to the elementary fields is included so that the action violates in general CP even with a single generation. This phase will not appear in the Higgs couplings (see end of section 3) but in more subtle observables such as correlation of masses. Following the literature we will take these IR parameters to be real in what follows, i.e. we consider a $\mathrm{CP}$ invariant composite sector.

In [1] we presented various scans for $\mathrm{CHM}_{5}$. There we used as a benchmark $f=$ $500 \mathrm{GeV}$ also in order to compare with results obtained in 5D models. This value of $f$ leads to a certain tension with electro-weak precision tests that typically requires new contributions (for example to the $T$-parameter) to agree with the data. Moreover for a light Higgs, as hinted by recent LHC data, the masses of the fermionic resonances are often lighter than $500 \mathrm{GeV}$ that is on the verge of being excluded by direct searches $[18,19]$. In this paper we present our results for the more conservative and perhaps more realistic choice $f=800 \mathrm{GeV}$ corresponding to a tuning parameter $\xi=v^{2} / f^{2} \simeq 0.1$, where precision tests are more easily satisfied. ${ }^{1}$ For this value the spin- 1 resonances can be easily above $3 \mathrm{TeV}$ so that contributions to the $S$-parameter are sufficiently suppressed and model dependent contributions to $T$ are typically within experimental bounds. In this case the resonances are heavier but could still be within the reach of the early LHC. The Higgs couplings are within $10 \%$ as in the SM so clearly our choice should be reconsidered if large deviations will be measured. Indeed this possibility is not yet excluded by present results [20-24].

As in our previous study we have performed a scan over the 6 fermionic parameters of the model, requiring that the correct electro-weak VEV and top mass are generated. ${ }^{2}$

\footnotetext{
${ }^{1}$ Our results can be easily extrapolated to different values of $f$. Neglecting higher order terms in $v^{2} / f^{2}$ and effects associated to the running of couplings (corresponding to higher loop corrections) we can rescale $f$ and all other dimensionful parameters of a given point and obtain in the vicinity of this point the electroweak VEV, approximately equal Higgs mass and fermion masses rescaled as $f$. This is because the Higgs mass is controlled by the dimensionless quartic coupling that is not rescaled. On the other hand the amount of cancellation of the quartic terms will grow proportionally to $f^{2}$.

${ }^{2}$ We demand $m_{t} \in[145,155]$ to roughly account for the running of the top mass which is generated at the scale of the heavy fermions $\sim \mathrm{TeV}$. A detailed analysis of the RGE is beyond the scope of our work. The spread on the top mass as chosen does not affect significantly the correlation between Higgs mass and
} 

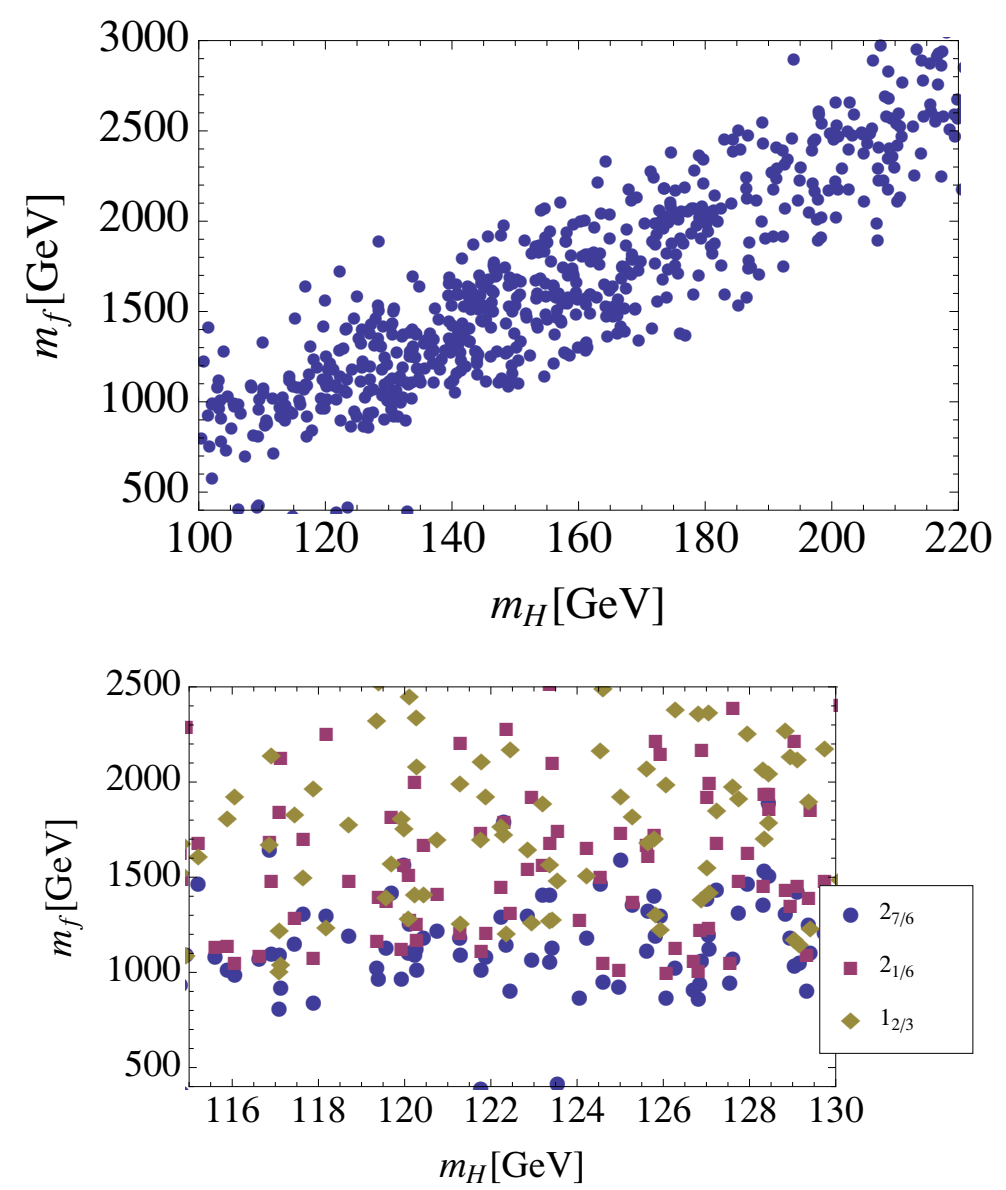

Figure 1. Masses of fermionic partners as a function of the Higgs mass for $f=800 \mathrm{GeV}$ in $\mathrm{CHM}_{5}$. The six fermionic parameters are varied between 0.3 and $4 \mathrm{TeV}$ and we require mixing elementary composite $\Delta_{t_{L}, t_{R}} / m_{T, \tilde{T}}<3$. The gauge contribution corresponds to $f_{1}=f_{2}=\sqrt{2} f$ and $g_{\rho}=3$. In the first panel we plot mass of the lightest fermionic partner as a function of the Higgs mass. In the second panel we plot mass of the fermionic excitations in the low mass region.

We have improved our numerical procedure in several ways compared to [1], including in particular all order corrections in $v / f$. Our plots are obtained using the standard approximation for the potential,

$$
V(h) \approx \alpha s_{h}^{2}-\beta s_{h}^{2} c_{h}^{2},
$$

where $s_{h}=\sin (h / f)$ and $h$ is the physical Higgs scalar. We have also performed the computations with the exact 1-loop Coleman-Weinberg effective potential finding that the corrections are negligible for $f=800 \mathrm{GeV}$ while they might be important for lower values of $f$. Requiring that the electro-weak VEV is reproduced one finds, ${ }^{3}$

$$
m_{h}^{2} \approx 8 \frac{\beta}{f^{4}} v^{2}
$$

\footnotetext{
mass of the resonances.

${ }^{3}$ The electro-weak VEV is defined as $v=f \sin (\langle h\rangle / f)$, where $v=246 \mathrm{GeV}$. From the form of the potential $s_{h}=\sqrt{(\beta-\alpha) / 2 \beta}$.
} 
which depends only on $\beta$. The coefficient $\alpha$ is proportional to the left or right mixings squared while $\beta$ is proportional to the top Yukawa squared. From the low energy point of view these contributions can be understood as generated from the loops of the Higgs dependent kinetic terms and of the top Yukawa respectively [1]. The natural size of $\beta$ is,

$$
\beta \approx \frac{N_{c} y_{t}^{2}}{16 \pi^{2}} f^{2} \Lambda^{2}
$$

where $\Lambda$ is the cut-off entering the top loops physically represented by the fermionic resonances. This is exactly reproduced in our model, see appendix B. From this the degree of tuning $m_{h}^{2} / \delta m_{h}^{2}$ scales as $v^{2} / f^{2}$. This can be considered as a lower bound on the tuning that is often larger because the typical size of $\alpha$ is larger than $\beta$.

The result of the scan is reported in figures 1 . In the first figure we show the correlation between the masses of the Higgs and the lightest fermionic resonances which in a large fraction of points is the doublet of hypercharge $7 / 6$ (the "custodian"), even though regions of parameters where the singlet is the lightest state can be found. Splittings generated by electro-weak symmetry breaking are neglected throughout. In the lower figure we zoom on the low Higgs mass region allowed by the LHC and show the mass of the singlet, doublet and custodian fermions.

A comment is in order. The natural size $\alpha$ is larger than $\beta$ so tuning the electro-weak

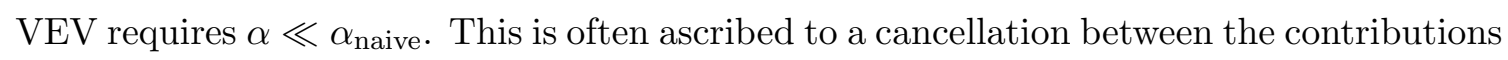
associated to the top left and top right. However it is also possible that each contribution is individually small. In our plots this happens in fraction of points of order $40 \%$. Here even the gauge contribution to $\alpha$ might be dominant, especially if the Higgs is light. We provide some analytical explanation of this fact in appendix B.

\subsubsection{Composite right-handed quarks}

In the anarchic composite scenarios, since symmetry breaking effects associated to the light generations are small, the potential is dominated by the loops of third generation quarks. In [26] it was however shown that right handed light quarks could be strongly composite as long as they couple to singlets of custodial symmetry as in the $\mathrm{CHM}_{5}$ model (see also $[27,28]$ for different realizations). In this case there are sizable contributions to the potential also from the light generations.

This latter scenario is strongly motivated by flavor as it allows the realization of Minimal Flavor Violation in CHM. This can be realized in $\mathrm{CHM}_{5}$ if the strong sector has a flavor symmetry $\mathrm{U}(3)_{U} \times \mathrm{U}(3)_{D}$ respected by the right-handed mixings,

$$
\Delta_{u_{R}}^{i j} \sim \delta^{i j}, \quad \Delta_{d_{R}}^{i j} \sim \delta^{i j} .
$$

so that the left-handed mixings are proportional to the SM Yukawas and are the only sources of breaking of the flavor symmetries. Since the right-handed mixings are equal by the flavor symmetry to the ones of the third generation, the contributions to the potential in particular from the up and charm quarks cannot be neglected.

The result of the scan is presented in figure 2 with the same parameters as in figure 1. Despite the new contributions to the potential we find a correlation between the Higgs mass and the mass of the lightest fermionic resonances similar to the one of the standard 

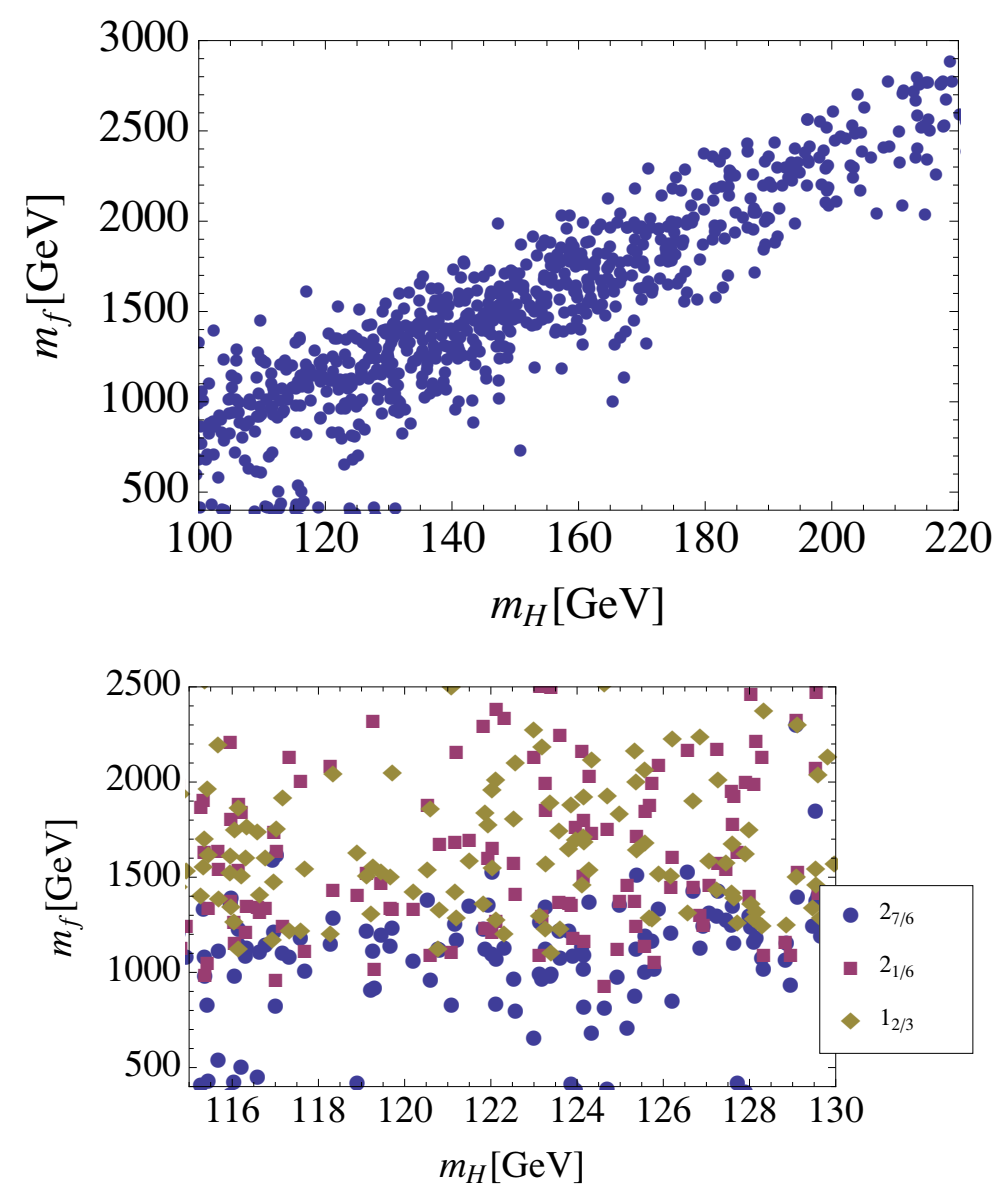

Figure 2. Masses of fermionic partners as a function of the Higgs mass for $f=800 \mathrm{GeV}$ in $\mathrm{CHM}_{5}$ with MFV. Same parameters as in figure 1 are chosen.

anarchic scenario. This can be easily understood. The light quarks only contribute to the coefficient $\alpha$ because their contribution to $\beta$ is small being proportional to the quark (mass) $^{2}$. After tuning the Higgs VEV, the Higgs mass only depends on $\beta$ (2.6) that is dominated by the top Yukawa. To realize this configuration however a different correlation between the left and right mixings is obtained.

\section{$2.3 \quad \mathrm{CHM}_{10}$}

Next we consider the model $\mathrm{CHM}_{10}$ where the composite fermions are in the $\mathbf{1 0}$ of $\mathrm{SO}(5)$. This was originally studied in [29] and in a different realization in [30]. Under $\mathrm{SU}(2)_{L} \times$ $\mathrm{SU}(2)_{R}$ the 10 decomposes as a $(\mathbf{2}, \mathbf{2}) \oplus(\mathbf{3}, \mathbf{1}) \oplus(\mathbf{1}, \mathbf{3})$. Each chiral SM fermion couples to a different $\mathbf{1 0}_{2 / 3}$ of $\mathrm{SO}(5) \times \mathrm{U}(1)_{X}$. The third generation quark lagrangian reads,

$$
\begin{aligned}
\mathcal{L}^{\mathrm{CHM}_{10}=} & \bar{q}_{L}^{e l} i \not D^{e l} q_{L}^{e l}+\bar{t}_{R}^{e l} i \not D^{e l} t_{R}^{e l} \\
& +\Delta_{t_{L}} \operatorname{Tr}\left[\bar{q}_{L} \Omega \Psi_{T}\right]+\Delta_{t_{R}} \operatorname{Tr}\left[\bar{t}_{R} \Omega \Psi_{\widetilde{T}}\right]+\text { h.c. } \\
& +\operatorname{Tr}\left[\bar{\Psi}_{T}\left(i \not D^{\rho}-m_{T}\right) \Psi_{T}\right]+\operatorname{Tr}\left[\bar{\Psi}_{\widetilde{T}}\left(i \not D^{\rho}-m_{\widetilde{T}}\right) \Psi_{\widetilde{T}}\right] \\
& -Y_{T} \Phi^{T} \bar{\Psi}_{T, L} \Psi_{\widetilde{T}, R} \Phi-m_{Y_{T}} \operatorname{Tr}\left[\bar{\Psi}_{T, L} \Psi_{\widetilde{T}, R}\right]+\text { h.c. }
\end{aligned}
$$



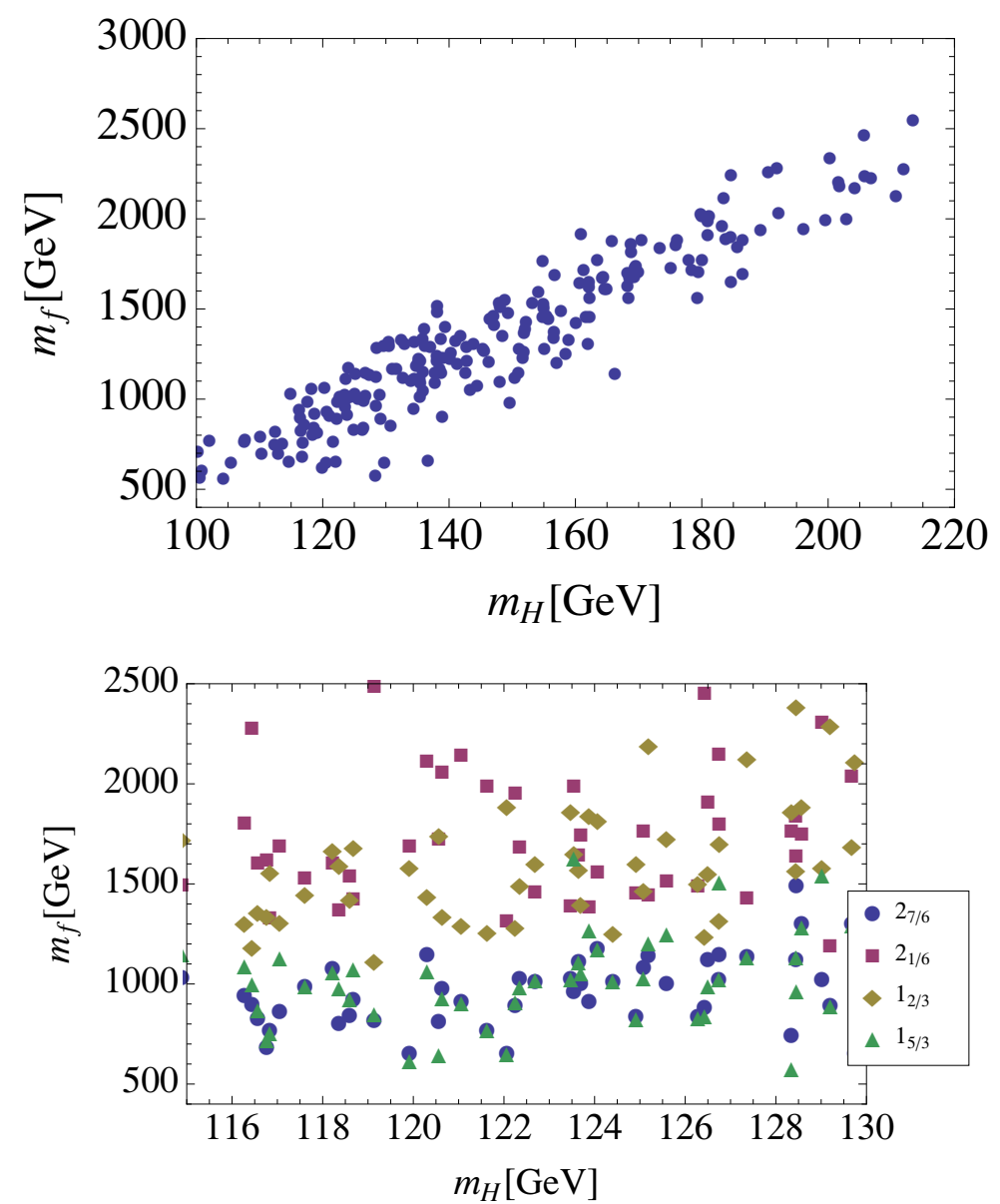

Figure 3. Higgs and fermionic partners masses in $\mathrm{CHM}_{10}$ for $f=800 \mathrm{GeV}$. The mass parameters are chosen (in TeV): $1<\Delta_{t_{L}, t_{R}}<5,0.5<m_{T, \tilde{T}}<3,-2<m_{Y_{T}}<.5$ and $3<Y_{T}<6$.

where $\Psi_{T, \tilde{T}}$ are $5 \times 5$ matrices corresponding to the adjoint rep of $\mathrm{SO}(5)$. The lagrangian has structure analogous to eq. (2.4), as an example we have the same LR chirality Yukawas in the strong sector, the presence of traces being needed to construct $\mathrm{SO}(5)$ invariants. Differently from $\mathrm{CHM}_{5}$ the SM doublet needs to couple to a single composite fermion to generate the Yukawas of up and down sector. As in $\mathrm{CHM}_{5}$ the latter is not important for the potential and we will neglect it.

We performed a scan of the potential similarly to $\mathrm{CHM}_{5}$ with $f=800 \mathrm{GeV}$. The main difference is due to the fact in $\mathrm{CHM}_{5}$ and $\mathrm{CHM}_{10}$ the $\mathrm{SM}$ fermions masses are related by,

$$
m_{q}^{\mathrm{CHM}_{10}} \sim \frac{1}{\sqrt{2}} m_{q}^{\mathrm{CHM}_{5}}
$$

for equal choice of parameters in the lagrangian. As a consequence a larger elementarycomposite mixings are typically required to reproduce the top mass. Moreover because of different group theory factors (see A.1.2) the size of the symmetry breaking coupling $Y_{T}$ needs to be chosen larger than in $\mathrm{CHM}_{5}$ to reproduce the electro-weak VEV and top mass. 
The global scan scan is shown in the upper plot in figure 3. In the lower plot we zoom in the low mass region. The fermionic spectrum is reacher than $\mathrm{CHM}_{5}$ containing a doublet $\mathbf{2}_{1 / 6}$ a singlet $\mathbf{1}_{2 / 3}$ a custodian $\mathbf{2}_{7 / 6}$ a triplet $\mathbf{3}_{2 / 3}$ and singlets $\mathbf{1}_{5 / 3}\left(\mathbf{1}_{-1 / 3}\right)$. The last three are degenerate up to electro-weak symmetry breaking effects. We note that in this model the first resonance tends to be lighter than in $\mathrm{CHM}_{5}$ while the states mixings with the SM fields are similar to that case. This is a consequence of the larger mixings required in this model that forces the states that do not couple to SM fields to be lighter.

\section{Coset space $\mathrm{SO}(6) / \mathrm{SO}(5)$}

The next simplest pattern of symmetry breaking relevant for $\mathrm{CH}$ is $\mathrm{SO}(6)$ broken to $\mathrm{SO}(5)$ originally introduced in [9]. There are 5 GBs transforming in the vectorial representation of $\mathrm{SO}(5)$, that decomposes into a doublet and a singlet under the electro-weak symmetry group. The coset space $\mathrm{SO}(6) / \mathrm{SO}(5)$ spanned by the GBs is the 5 -sphere that can be conveniently parametrized by a unit vector of $\mathrm{SO}(6)$,

$$
\Phi=\sin \frac{\varphi}{f}\left(\frac{h_{1}}{\varphi}, \frac{h_{2}}{\varphi}, \frac{h_{3}}{\varphi}, \frac{h_{4}}{\varphi}, \frac{s}{\varphi}, \cot \frac{\varphi}{f}\right), \quad \varphi=\sqrt{\vec{h}^{2}+s^{2}}
$$

After electro-weak symmetry breaking one can choose the unitary gauge $h_{1}=h_{2}=h_{3}=0$ so that the physical degrees of freedom are $h=h_{4}$ and the singlet $s$. The following parametrization will be convenient,

$$
\begin{aligned}
& h=\varphi \cos \frac{\psi}{f} \\
& s=\varphi \sin \frac{\psi}{f}
\end{aligned} \quad \rightarrow \quad \Phi=\left(0,0,0, \sin \frac{\varphi}{f} \cos \frac{\psi}{f}, \sin \frac{\varphi}{f} \sin \frac{\psi}{f}, \cos \frac{\varphi}{f}\right)
$$

where $\varphi$ is a scalar and $\psi$ a pseudo-scalar under CP. The kinetic terms correspond to a 2-sphere with standard metric,

$$
d s^{2}=d \varphi^{2}+\sin ^{2} \frac{\varphi}{f} d \psi^{2}
$$

\subsection{Gauge sector}

The gauge lagrangian has the same structure as eq. (2.1). The low energy action for the Higgs and gauge fields is given by,

$$
\frac{f^{2}}{2}\left(D_{\mu} \Phi\right)^{T}\left(D^{\mu} \Phi\right)=\frac{1}{2}(\partial \varphi)^{2}+\frac{1}{2} \sin ^{2} \frac{\varphi}{f}(\partial \psi)^{2}+\frac{g^{2} f^{2}}{4} \sin ^{2} \frac{\varphi}{f} \cos ^{2} \frac{\psi}{f} W^{2}+\cdots
$$

from which we identify the electro-weak VEV as,

$$
v=f \sin \frac{\varphi}{f} \cos \frac{\psi}{f}
$$

As far as the gauge sector is concerned, on any vacuum one can always define a CP symmetry that is preserved. 


\subsection{Fermionic sector}

The simplest option for the composite fermions is that they are in a vector representation of $\mathrm{SO}(6)$ and for simplicity we will focus on this here. The fermionic action has the same structure as eq. (2.4). The embedding of the up quarks in the 6 reads

$$
q_{L} \rightarrow \frac{1}{\sqrt{2}}\left(\begin{array}{c}
b_{L} \\
-i b_{L} \\
t_{L} \\
i t_{L} \\
0 \\
0
\end{array}\right), \quad t_{R} \rightarrow\left(\begin{array}{c}
0 \\
0 \\
0 \\
0 \\
e^{i \delta} \cos \theta t_{R} \\
\sin \theta t_{R}
\end{array}\right)
$$

and similarly for the down quarks. Note that $t_{R}$ can be coupled both to the fifth or sixth component of the vector, with complex phase $\delta$. Since the action for the composite fields is (non-linearly) invariant under $\mathrm{SO}(6)$, through an $\mathrm{SO}(2)$ rotation of the fifth and sixth component (under which the $\mathrm{SO}(4)$ generators do not transform and the singlet shifts) and a phase redefinition of $t_{R}$ one can choose a basis where $\delta=\pi / 2$. In this basis it is manifest that the couplings of the top respects the CP symmetry [10],

$$
\Psi \rightarrow \Omega_{0}\left(-i \gamma_{2} \gamma_{0}\right) \Psi^{*}, \quad \Omega_{0}=\operatorname{Diag}[1,-1,1,-1,-1,1]
$$

As in the $\mathrm{CHM}_{5} \mathrm{CP}$ could be explicitly broken by the phases of the strong sector but we will not consider this possibility here.

There are two special values of $\theta$. For $\theta=\pi / 4$ the mixing of $t_{R}$ preserves the $\mathrm{SO}(2)$ subgroup of $\mathrm{SO}(6)$ which rotates the fifth and sixth component, simultaneously with a phase transformation of $t_{R}$. In this limit the singlet is an exact GB and it will remain massless if the only contribution to the potential is due to coupling to SM quarks. ${ }^{4} \theta=\pi / 2$ is also special because the mixings leave an unbroken $Z_{2}$ symmetry under which $\psi$ changes sign. This guarantees the stability of the pseudo-scalar that could be used as dark matter candidate [31].

\subsection{Higgs and singlet mass}

Let us now turn to the potential. Obviously the $\mathrm{CP}$ invariant vacuum $\psi=0$ is an extremum of the potential. If $\psi$ acquires a $\mathrm{VEV}$ because $\psi=0$ is a maximum one might think that $\mathrm{CP}$ is spontaneously broken. This is not the case however, at least in the limit where only the contribution of the top is included, see appendix A.2. Inspecting the potential one can prove that if $\psi=0$ is a maximum, the minimum of the potential corresponds to,

$$
\sin \frac{\varphi}{f}=1 .
$$

In this vacuum the role of the fluctuations of $\psi$ and $\varphi$ are exchanged and a different definition of $\mathrm{CP}$ exists that leaves the vacuum invariant. One can check that this vacuum is

\footnotetext{
${ }^{4} \mathrm{~A}$ tiny mass would be generated at 2-loops through anomalies with SM fields. In this case the singlet would be an electro-weak axion ruled out experimentally [9].
} 

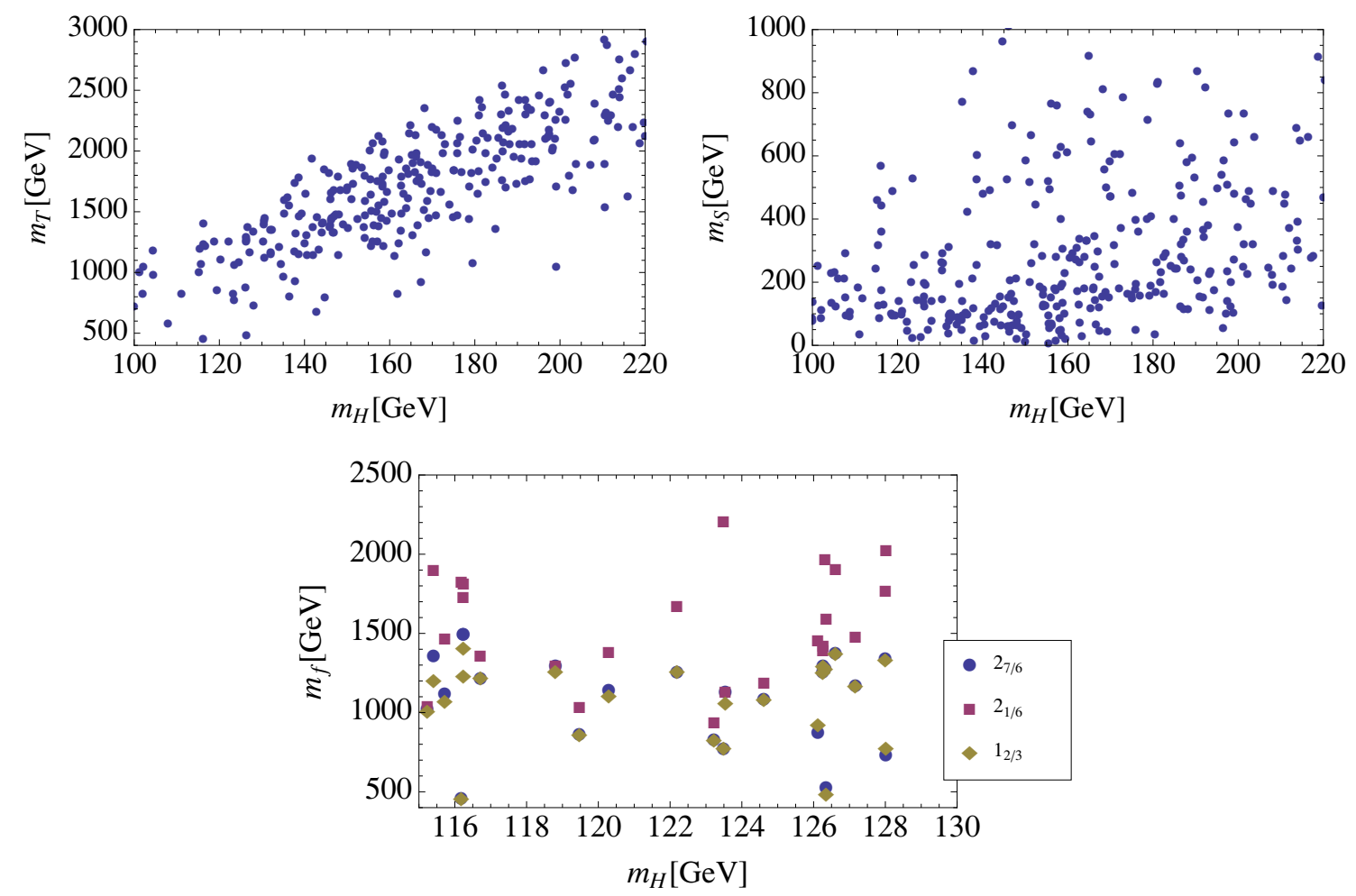

Figure 4. Masses of Higgs, singlet and fermionic partners in $\mathrm{CHM}_{6}$ for $f=800 \mathrm{GeV}$. The six fermionic parameters are varied between 0.3 and $4 \mathrm{TeV}$ with the mixing elementary-composite $\Delta_{t_{L}, t_{R}} / m_{T, \tilde{T}}<3$. The gauge contribution corresponds to $f_{1}=f_{2}=\sqrt{2} f$ and $g_{\rho}=3$. Upper left panel: Higgs mass vs. mass of lightest fermionic state. Upper right panel: Higgs mass vs. singlet mass. Below masses of lightest singlet, doublet and custodian in the low Higgs mass region.

physically equivalent to the one with $\psi=0$ so without loss of generality we will assume this configuration to be realized. We conclude that $\mathrm{CP}$ can be neither explicitly nor spontaneously broken by the coupling to the top. A small breaking of CP could be induced from the contributions to the potential of the lighter generations neglected here.

The computation of the potential is similar to $\mathrm{CHM}_{5}$, some details are in appendix A. As far as Higgs mass is concerned the formulas are identical to that case, the main difference being that in $\mathrm{SO}(6) / \mathrm{SO}(5)$, for equal choice of parameters one finds,

$$
m_{q}^{\mathrm{CHM}_{6}} \sim m_{q}^{\mathrm{CHM}_{5}} \sin \theta .
$$

This means that to realize the top mass larger mixings of the elementary fields are necessary than in $\mathrm{CHM}_{5}$, unless $\theta \sim \pi / 2$.

We performed an unconstrained scan over the parameters of the model including the angle $\theta$. The range of parameters is chosen as in $\mathrm{CHM}_{5}$. In first of figures 4 we present a scan of points of parameter space with the correct EWSB and top mass for $f=800$. We find roughly the same correlation between the Higgs mass and the mass of the lightest fermionic partners as in the $\mathrm{SO}(5) / \mathrm{SO}(4)$ model. This is expected because the Higgs mass is controlled by $y_{t}^{2}$. The larger spread of masses can be understood as due to fact that 

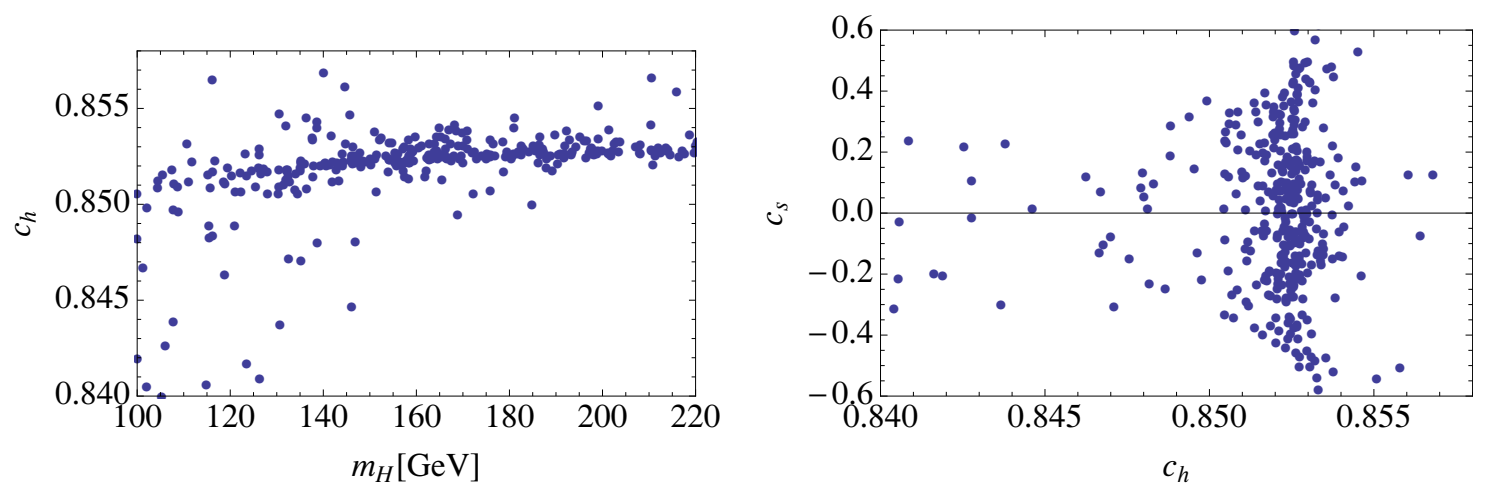

Figure 5. Coupling of the scalars to fermions including wave-function normalization effects. On the left Higgs coupling to the top vs. Higgs mass. On the right the Higgs vs. singlet coupling.

larger mixings are necessary if $\theta<\pi / 2$. Note that the lightest state in this case is often a singlet. This can be understood from the fact that contrary to $\mathrm{CHM}_{5}$ one fermion singlet combination does not mix with the SM fields so its mass is lighter as in the case of the $\mathbf{2}_{7 / 6}$ doublet in $\mathrm{CHM}_{5}$.

The mass of the CP odd singlet is typically heavier than the one of the Higgs. This is shown in second figure 4 . This agrees with the fact that while the Higgs mass is controlled by the tuned electro-weak VEV the mass of the singlet follows naturalness. However, if $\theta$ is close to $\pi / 4$ the singlet becomes an approximate GB and small values of the mass are then obtained. For $\theta=\pi / 2$ the Higgs mass is identical to $\mathrm{CHM}_{5}$ while the singlet is heavy.

\subsection{Modified couplings}

The low energy interactions of the Higgs and singlet to gauge fields and fermions can be parametrized similarly to ref. [25] as,

$$
\begin{aligned}
\mathcal{L}= & \frac{1}{2}\left(\partial_{\mu} h\right)^{2}+\frac{1}{2}\left(\partial_{\mu} s\right)^{2}-V(h, s)+\frac{v^{2}}{4} \operatorname{Tr}\left(D_{\mu} \Sigma^{\dagger} D^{\mu} \Sigma\right)\left[1+2 a_{h} \frac{h}{v}+b_{h} \frac{h^{2}}{v^{2}}+b_{s} \frac{s^{2}}{v^{2}} \ldots\right] \\
& -m_{i} \bar{\psi}_{L i} \Sigma\left(1+c_{h} \frac{h}{v}+\ldots\right) \psi_{R i}-m_{i} \bar{\psi}_{L i}\left(c_{s} \frac{s}{v}+\ldots\right) \psi_{R i}+\text { h.c. }
\end{aligned}
$$

where $\Sigma=\exp \left(i \chi^{a} \sigma^{a} / v\right)$ contains the GBs eaten by $W$ and $Z$ bosons and we suppressed terms that violate $\mathrm{CP}$ since the vacuum we are considering respects the symmetry.

The couplings of $a$ and $b$ are obtained expanding eq. (3.5) around the vacuum and taking into account the kinetic terms (3.3),

$$
a_{h}=\sqrt{1-\xi}, \quad b_{h}=1-2 \xi, \quad b_{s}=1
$$

The coupling to fermions can be extracted from the effective action in A.2 that contains,

$$
\frac{M_{1}^{u}(0)}{\sqrt{\Pi_{t_{L}}(0) \Pi_{t_{R}}(0)}} \bar{t}_{L} t_{R} s_{\varphi} c_{\psi}\left(i c_{\theta} s_{\varphi} s_{\psi}+s_{\theta} c_{\varphi}\right)+\text { h.c. }
$$


where we abbreviate $\sin x / f \equiv s_{x}$. Neglecting wave-function normalization effects (arising from the Higgs dependence in $\Pi_{t_{L}}$ and $\Pi_{t_{R}}$ ) we find,

$$
c_{h}=\frac{1-2 \xi}{\sqrt{1-\xi}} \quad c_{s}=i \sqrt{\frac{\xi}{1-\xi}} \cot \theta
$$

In figure 5 we compute the exact couplings to the fermions for the points of figure 4 (for $f=800 \mathrm{GeV}$ we get $\left.c_{h} \simeq 0.85\right)$. On the left we see that on the point that realize the $\mathrm{SM}$ vacuum the effect of wave-function normalization is small even for a light Higgs where some light partners exist. There is no correlation between the singlet coupling and the Higgs mass.

The coupling of the singlet to fermions could be in principle larger than the SM Higgs coupling, however this requires a small value of $\theta$ where it is more difficult to obtain the top mass. Indeed, as shown in the second figure 5 , in our sample it is always smaller. The couplings of the singlet vanish for $\theta=\pi / 2$ as required by the $Z_{2}$ symmetry $\psi \rightarrow-\psi$. Note that a possible phase in $M_{1}^{u}$, arising if the IR parameters $Y_{T}$ and $M_{Y_{T}}$ are complex does not affect the couplings since it is reabsorbed into the top mass. This shows that the CP violating phase of the strong sector does not induce $\mathrm{CP}$ violation in the scalar couplings.

At loop level the Higgs and singlet will also couple SM gauge bosons. For gluons we parametrize,

$$
\mathcal{L}_{g g h}=\frac{\alpha_{s}}{12 \pi} g_{h G G} \frac{h}{v} G_{\mu \nu}^{a} G_{\mu \nu}^{a}, \quad \mathcal{L}_{g g s}=\frac{\alpha_{s}}{16 \pi} g_{s G G} \frac{s}{v} G_{\mu \nu}^{a} \tilde{G}_{\mu \nu}^{a}
$$

where $\tilde{G}_{\mu \nu}^{a}=\epsilon_{\mu \nu \rho \sigma} G^{a \rho \sigma}$. In the limit where Higgs and singlet are lighter than the top but heavier than light quarks the coupling is determined by the top couplings above. Following [32] we derive,

$$
\begin{aligned}
& g_{h G G}=\left.v \frac{\partial}{\partial \varphi} \log \operatorname{det} \mathcal{M}(\varphi, \psi)\right|_{\mathrm{VEV}}=\frac{1-2 \xi}{\sqrt{1-\xi}} \\
& g_{s G G}=\left.v \frac{\partial}{\partial \psi} \log \operatorname{det} \mathcal{M}(\varphi, \psi)\right|_{\mathrm{VEV}}=\sqrt{\frac{\xi}{1-\xi}} \cot \theta
\end{aligned}
$$

where $\mathcal{M}(\varphi, \psi)$ is the fermionic mass matrix. The coupling of the Higgs is the same as in $\mathrm{CHM}_{5}$. The coupling of the singlet breaks the shift symmetry but does not vanish when $\theta=\pi / 4$ where the Goldstone symmetry is recovered. This is due to fact that the $\mathrm{U}(1)$ rotation of $t_{R}$ is anomalous.

\section{Discussion}

In this note we have extended the study of the Higgs mass in composite Higgs models with partial compositeness initiated in ref. [1]. We have considered different fermionic setups for $\mathrm{SO}(5)$ and studied also the potential for $\mathrm{SO}(6) / \mathrm{SO}(5)$ where an extra $\mathrm{CP}$ odd scalar is present in the spectrum. Along the way we have also clarified various $\mathrm{CP}$ properties of these theories. For example in $\mathrm{SO}(6) / \mathrm{SO}(5) \mathrm{CP}$ can neither be explicitly nor spontaneously 


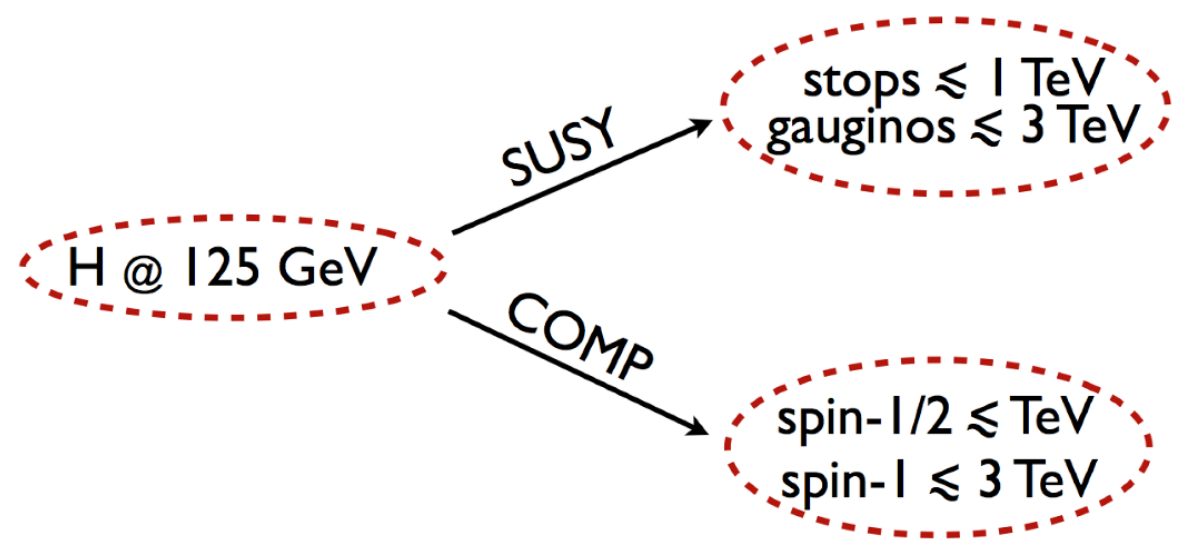

Figure 6. Schematic picture for the expected natural scenarios in CHM and SUSY.

broken by the couplings of the top so we expect the coupling of the singlet to be CP preserving.

The main result is that a light Higgs, hinted by recent experimental results, typically requires fermionic partners lighter than $\mathrm{TeV}$. This represents a great opportunity for the LHC that will be able to discover or exclude these fermionic partners in the early stages of the $14 \mathrm{TeV}$ run, or even in the present run under favorable circumstances. While not a general property of CHM in all the models considered, from eqs. (2.6), (2.7) the Higgs mass scales as,

$$
m_{h} \sim \sqrt{\frac{N_{c}}{2}} \frac{y_{t}}{\pi} \frac{\Lambda}{f} v
$$

where $\Lambda$ is the cutoff of the quadratic divergence associated to the top Yukawa $y_{t}$. The cutoff is physically represented by the lighter resonances, though not necessarily the lightest. The necessity of light fermionic states can be understood in a model independent way from naturalness of the theory. With a light Higgs, the unavoidable quadratic divergence of the Higgs mass generated by the top Yukawa must be cut-off at a scale around $\mathrm{TeV}$ if a tuning of order $10 \%$ is allowed (measured as $v^{2} / f^{2}$ ). This demands new fermionic states to saturate the Higgs potential at a scale lower than spin-1 resonances. In composite Higgs models other contributions to the potential exist but at least with the fermionic representations considered here the Higgs mass is determined by the loops associated with the top Yukawa. If contributions larger than that control the Higgs mass, naturalness would require even lighter states. Therefore our estimates on the mass of the resonances should be considered as an upper bound.

We can draw a parallel with supersymmetry as described in figure 6. In supersymmetry the naturalness of the electro-weak scale requires most minimally light stops (below $\mathrm{TeV}$ ) and possibly heavier winos and binos to cut-off the gauge loops, while other partners could be heavier and even beyond the LHC reach. This spectrum, dubbed natural SUSY, is to date the most compelling scenario for supersymmetry [33, 34]. Bounds on light stops will soon reach $\sim 500 \mathrm{GeV}$, a figure similar to the bound on fermionic partners in composite 
models. If naturalness is a good guide, either new colored scalars or colored fermions should be soon discovered at the LHC.

\section{Acknowledgments}

We are grateful to Stefania De Curtis for collaboration on related subjects. We would like to thank Roberto Contino, Giuliano Panico, Riccardo Rattazzi and Andrea Wulzer for stimulating discussions. We also thank GP and AW for sharing with us their results [35] before publication.

\section{A Basic formulas}

In this appendix we collect the basic formulas of the models studied in this paper. More details can be found in [1].

For the gauge sector we have, up to electro-weak symmetry breaking effects,

$$
\begin{array}{ll}
m_{\rho}^{2}=\frac{g_{\rho}^{2} f_{1}^{2}}{2}, & m_{a_{1}}^{2}=\frac{g_{\rho}^{2}\left(f_{1}^{2}+f_{2}^{2}\right)}{2}, \quad f^{2}=\frac{f_{1}^{2} f_{2}^{2}}{f_{1}^{2}+f_{2}^{2}} \\
\frac{1}{g^{2}}=\frac{1}{g_{0}^{2}}+\frac{1}{g_{\rho}^{2}}, & \frac{1}{g^{\prime 2}}=\frac{1}{g_{0 Y}^{2}}+\frac{1}{g_{\rho}^{2}}+\frac{1}{g_{\rho_{X}}^{2}},
\end{array}
$$

where $f$ is the decay constant of the GB Higgs and $g, g^{\prime}$ are the SM couplings. $m_{\rho}$ and $m_{a_{1}}$ are the masses of resonances of $\mathrm{SO}(4)$ and $\mathrm{SO}(5) / \mathrm{SO}(4)$, while $g_{\rho}$ and $g_{\rho_{X}}$ are the couplings of the composite resonances of $\mathrm{SO}(5)$ and $\mathrm{U}(1)_{X}$. The mass of $\rho_{X}$ is a free parameter.

\section{A.1 $\mathrm{SO}(5) / \mathrm{SO}(4)$}

The effective action for $\mathrm{SM}$ fermions in $\mathrm{CHM}_{5}$ and $\mathrm{CHM}_{10}$ obtained integrating out the strong sector takes the form [29],

$$
\begin{aligned}
\mathcal{L}_{\text {eff }}= & \bar{q}_{L} \not p\left[\Pi_{0}^{q}\left(p^{2}\right)+\frac{s_{h}^{2}}{2}\left(\Pi_{1}^{q 1}\left(p^{2}\right) \widehat{H}^{c} \widehat{H}^{c \dagger}+\Pi_{1}^{q 2}\left(p^{2}\right) \widehat{H} \widehat{H}^{\dagger}\right)\right] q_{L} \\
& +\bar{u}_{R} \not p\left(\Pi_{0}^{u}\left(p^{2}\right)+\frac{s_{h}^{2}}{2} \Pi_{1}^{u}\left(p^{2}\right)\right) u_{R}+\bar{d}_{R} \not p\left(\Pi_{0}^{d}\left(p^{2}\right)+\frac{s_{h}^{2}}{2} \Pi_{1}^{d}\left(p^{2}\right)\right) d_{R} \\
& +\frac{s_{h} c_{h}}{\sqrt{2}} M_{1}^{u}\left(p^{2}\right) \bar{q}_{L} \widehat{H}^{c} u_{R}+\frac{s_{h} c_{h}}{\sqrt{2}} M_{1}^{d}\left(p^{2}\right) \bar{q}_{L} \widehat{H} d_{R}+\text { h.c. }
\end{aligned}
$$

The 1-loop effective potential due to the top loop is given by,

$$
V(h)_{\text {fermions }}=-2 N_{c} \int \frac{d^{4} p}{(2 \pi)^{4}}\left[\ln \Pi_{b_{L}}+\ln \left(p^{2} \Pi_{t_{L}} \Pi_{t_{R}}-\Pi_{t_{L} t_{R}}^{2}\right)\right]
$$

where,

$$
\begin{aligned}
\Pi_{t_{L}} & =\Pi_{0}^{q}+\frac{\sin ^{2}(h / f)}{2} \Pi_{1}^{q_{1}}, & \Pi_{b_{L}} & =\Pi_{0}^{q}+\frac{\sin ^{2}(h / f)}{2} \Pi_{1}^{q_{2}} \\
\Pi_{t_{R}} & =\Pi_{0}^{u}+\frac{\sin ^{2}(h / f)}{2} \Pi_{1}^{u}, & \Pi_{t_{L} t_{R}} & =\frac{\sin (h / f) \cos (h / f)}{\sqrt{2}} M_{1}^{u} .
\end{aligned}
$$


For $s_{h} \ll 1$ one can approximate,

$$
V(h)_{\mathrm{fermions}} \approx \alpha s_{h}^{2}-\beta s_{h}^{2} c_{h}^{2}
$$

where

$$
\begin{aligned}
& \alpha=-N_{c} \int \frac{d^{4} p}{(2 \pi)^{4}}\left[\frac{\Pi_{1}^{q_{1}}}{\Pi_{0}^{q}}+\frac{\Pi_{1}^{u}}{\Pi_{0}^{u}}\right] \\
& \beta=-N_{c} \int \frac{d^{4} p}{(2 \pi)^{4}} \frac{\left(M_{1}^{u}\right)^{2}}{p^{2}\left(\Pi_{0}^{q}+s_{h}^{2} / 2 \Pi_{1}^{q_{1}}\right)\left(\Pi_{0}^{u}+s_{h}^{2} / 2 \Pi_{1}^{u}\right)}
\end{aligned}
$$

The gauge loops also contribute to $\alpha$.

In our model the form factors can be expressed in terms of the functions,

$$
\begin{aligned}
\widehat{\Pi}\left[m_{1}, m_{2}, m_{3}\right] & =\frac{\left(m_{2}^{2}+m_{3}^{2}-p^{2}\right) \Delta^{2}}{p^{4}-p^{2}\left(m_{1}^{2}+m_{2}^{2}+m_{3}^{2}\right)+m_{1}^{2} m_{2}^{2}}, \\
\widehat{M}\left[m_{1}, m_{2}, m_{3}\right] & =-\frac{m_{1} m_{2} m_{3} \Delta^{2}}{p^{4}-p^{2}\left(m_{1}^{2}+m_{2}^{2}+m_{3}^{2}\right)+m_{1}^{2} m_{2}^{2}} .
\end{aligned}
$$

as follows:

\section{A.1.1 $\mathrm{CHM}_{5}$}

Including only the top the form factors are given by,

$$
\begin{array}{rlrl}
\Pi_{0}^{q} & =\frac{1}{y_{t_{L}}^{2}}+\widehat{\Pi}_{0}^{q_{L}}, & \Pi_{1}^{q_{1}} & =\widehat{\Pi}_{1}^{q_{L}}, \\
\Pi_{0}^{u}=\frac{1}{y_{t_{R}}^{2}}+\widehat{\Pi}_{0}^{u_{R}}+\widehat{\Pi}_{1}^{u_{R}}, & \Pi_{1}^{q_{2}}=0, & \\
\Pi_{1}^{u} & =-2 \widehat{\Pi}_{1}^{u_{R}} &
\end{array} \quad M_{1}^{u}=\widehat{M}_{1}^{u},
$$

where

$$
\begin{aligned}
\widehat{\Pi}_{0}^{q_{L}} & =\widehat{\Pi}\left[m_{T}, m_{\widetilde{T}}, m_{Y_{T}}\right], & \widehat{\Pi}_{1}^{q_{L}} & =\widehat{\Pi}\left[m_{T}, m_{\widetilde{T}}, m_{Y_{T}}+Y_{T}\right]-\widehat{\Pi}\left[m_{T}, m_{\widetilde{T}}, m_{Y_{T}}\right], \\
\widehat{\Pi}_{0}^{u_{R}} & =\widehat{\Pi}\left[m_{\widetilde{T}}, m_{T}, m_{Y_{T}}\right], & \widehat{\Pi}_{1}^{u_{R}} & =\widehat{\Pi}\left[m_{\widetilde{T}}, m_{T}, m_{Y_{T}}+Y_{T}\right]-\widehat{\Pi}\left[m_{\widetilde{T}}, m_{T}, m_{Y_{T}}\right], \\
\widehat{M}_{0}^{u} & =\widehat{M}\left[m_{T}, m_{\widetilde{T}}, m_{Y_{T}}\right], & \widehat{M_{1}^{u}} & =\widehat{\Pi}\left[m_{T}, m_{\widetilde{T}}, m_{Y_{T}}+Y_{T}\right]-\widehat{\Pi}\left[m_{T}, m_{\widetilde{T}}, m_{Y_{T}}\right] .
\end{aligned}
$$

and to match with the lagrangian (2.4) $\Delta_{t_{L}, t_{R}}=y_{t_{L}, t_{R}} \Delta$.

Up to electro-weak symmetry breaking effects the masses of the $\mathbf{2}_{\mathbf{1} / \mathbf{6}}, \mathbf{2}_{\mathbf{7}, \mathbf{6}}$ and $\mathbf{1}_{\mathbf{2} / \mathbf{3}}$ fermions are given by the zeros of $\Pi_{0}^{q}$, the poles of $\Pi_{0}^{q}$ and the zeros of $\Pi_{0}^{u}$ respectively.

\section{A.1.2 $\mathrm{CHM}_{10}$}

In this case one finds,

$$
\begin{array}{rlrl}
\Pi_{0}^{q} & =\frac{1}{y_{t_{L}}^{2}}+\widehat{\Pi}_{0}^{q_{L}}+\frac{1}{2} \widehat{\Pi}_{1}^{q_{L}}, & \Pi_{1}^{q_{1}} & =-\frac{1}{2} \widehat{\Pi}_{1}^{q_{L}}, \\
\Pi_{0}^{u}=\frac{1}{y_{t_{R}}^{2}}+\widehat{\Pi}_{0}^{u_{R}}, & \Pi_{1}^{q_{2}}=-\widehat{\Pi}_{1}^{q_{L}}, & M_{1}^{u}=\frac{1}{2 \sqrt{2}} \widehat{M}_{1}^{u}, \\
\Pi_{1}^{u}=\frac{1}{2} \widehat{\Pi}_{1}^{u_{R}}, &
\end{array}
$$


where

$$
\begin{aligned}
\widehat{\Pi}_{0}^{q_{L}} & =\widehat{\Pi}\left[m_{T}, m_{\widetilde{T}}, m_{Y_{T}}\right], & \widehat{\Pi}_{1}^{q_{L}} & =2 \widehat{\Pi}\left[m_{T}, m_{\widetilde{T}}, m_{Y_{T}}+Y_{T} / 2\right]-2 \widehat{\Pi}\left[m_{T}, m_{\widetilde{T}}, m_{Y_{T}}\right], \\
\widehat{\Pi}_{0}^{u_{R}} & =\widehat{\Pi}\left[m_{\widetilde{T}}, m_{T}, m_{Y_{T}}\right], & \widehat{\Pi}_{1}^{u_{R}} & =2 \widehat{\Pi}\left[m_{\widetilde{T}}, m_{T}, m_{Y_{T}}+Y_{T} / 2\right]-2 \widehat{\Pi}\left[m_{\widetilde{T}}, m_{T}, m_{Y_{T}}\right], \\
\widehat{M}_{0}^{u} & =\widehat{M}\left[m_{T}, m_{\widetilde{T}}, m_{Y_{T}}\right], & \widehat{M}_{1}^{u} & =2 \widehat{\Pi}\left[m_{T}, m_{\widetilde{T}}, m_{Y_{T}}+Y_{T} / 2\right]-2 \widehat{\Pi}\left[m_{T}, m_{\widetilde{T}}, m_{Y_{T}}\right] .
\end{aligned}
$$

The masses of the $\mathbf{2}_{\mathbf{1 / 6}}, \mathbf{2}_{\mathbf{7 , 6}} \mathbf{1}_{\mathbf{2} / \mathbf{3}}$ and $\left(\mathbf{1}_{\mathbf{5} / \mathbf{3}}, \mathbf{1}_{-\mathbf{1} / \mathbf{3}}, \mathbf{3}_{\mathbf{2} / \mathbf{3}}\right)$ fermions are given by the zeros of $\Pi_{0}^{q}$, the poles of $\Pi_{0}^{q}$, the zeros of $\Pi_{0}^{u}$ and the poles $\Pi_{0}^{u}$ respectively.

\section{$\mathrm{A} .2 \mathrm{SO}(6) / \mathrm{SO}(5)$}

With composite fermions in the $\mathbf{6}$ the effective lagrangian for the SM fermions reads,

$$
\begin{aligned}
\mathcal{L}_{\text {eff }}= & \bar{q}_{L} \not p\left(\widehat{\Pi}_{0}^{q_{L}}(p)+\frac{1}{2} \frac{s_{\varphi}^{2}}{\varphi^{2}} \widehat{\Pi}_{1}^{q_{L}}(p) H^{c} H^{c}\right) q_{L} \\
& +\bar{u}_{R \not p}\left(\widehat{\Pi}_{0}^{u_{R}}(p)+s_{\theta}^{2} \widehat{\Pi}_{1}^{u_{R}}(p)+\left[s_{\varphi}^{2}\left(c_{\theta}^{2} s_{\psi}^{2}-s_{\theta}^{2}\right)\right] \widehat{\Pi}_{1}^{u_{R}}(p)\right) u_{R} \\
& +\frac{\widehat{M}_{1}^{u}(p)}{\sqrt{2}} \frac{s_{\varphi}}{\varphi} \bar{q}_{L} H^{c}\left(i c_{\theta} s_{\varphi} s_{\psi}+s_{\theta} c_{\varphi}\right) u_{R}+\text { h.c. }
\end{aligned}
$$

where the form factors are,

$$
\begin{aligned}
\Pi_{0}^{q} & =\frac{1}{y_{t_{L}}^{2}}+\widehat{\Pi}_{0}^{q_{L}}, & \Pi_{1}^{q_{1}} & =\widehat{\Pi}_{1}^{q_{L}}, \\
\Pi_{0}^{u}=\frac{1}{y_{t_{R}}^{2}}+\widehat{\Pi}_{0}^{u_{R}}+s_{\theta}^{2} \widehat{\Pi}_{1}^{u_{R}}, & \Pi_{1}^{q_{2}}=0, & \Pi_{1}^{u}=-2 \widehat{\Pi}_{1}^{u_{R}} &
\end{aligned} M_{1}^{u}=\widehat{M}_{1}^{u},
$$

in terms of the building blocks (A.9).

The potential has now the form,

$$
\begin{aligned}
V(\varphi, \psi) \simeq & c_{1} \sin ^{2} \varphi \cos ^{2} \psi+c_{2} \sin ^{2} \varphi\left(\sin ^{2} \theta-\cos ^{2} \theta \sin ^{2} \psi\right) \\
& -c_{3} \sin ^{2} \varphi \cos ^{2} \psi\left[\cos ^{2} \theta \sin ^{2} \varphi \sin ^{2} \psi+\sin ^{2} \theta \cos ^{2} \varphi\right]
\end{aligned}
$$

where,

$$
\begin{aligned}
c_{1} & =-N_{c} \int \frac{d^{4} p}{(2 \pi)^{4}} \frac{\Pi_{1}^{q_{1}}}{\Pi_{0}^{q}}, \quad c_{2}=-N_{c} \int \frac{d^{4} p}{(2 \pi)^{4}} \frac{\Pi_{1}^{u}}{\Pi_{0}^{u}}, \\
c_{3} & =-N_{c} \int \frac{d^{4} p}{(2 \pi)^{4}} \frac{\left(M_{1}^{u}\right)^{2}}{\left(\Pi_{0}^{q}+s_{h}^{2} \Pi_{1}^{q_{1}} / 2\right)\left(\Pi_{0}^{u}+s_{h}^{2} s_{\theta}^{2} \Pi_{1}^{u} / 2\right)}
\end{aligned}
$$

The gauge loops also contribute to the coefficient $c_{1}$. Two extrema of the potential are $\psi=0$ and $s_{\varphi}=1$. One can prove that other extrema of the potential are saddle points so that one is the minimum while the other is the maximum. Note that since the lagrangian is invariant under the transformations,

$$
\begin{aligned}
s_{\varphi} s_{\psi} & \rightarrow c_{\varphi}^{\prime} \\
c_{\varphi} & \rightarrow s_{\varphi}^{\prime} s_{\psi}^{\prime} \\
s_{\theta} & \rightarrow c_{\theta}
\end{aligned}
$$


one can choose without loss of generality the minimum at $\psi=0$. The Higgs and singlet mass are then given by,

$$
\begin{aligned}
& m_{h}^{2}=\frac{-4 c_{1} c_{2}-2 c_{1}^{2} / s_{\theta}^{2}+2\left(c_{3}^{2}-c_{2}^{2}\right) s_{\theta}^{2}}{c_{3} f^{2}} \\
& m_{s}^{2}=\frac{c_{1}-\left(c_{2}+c_{3}\right) s_{\theta}^{2}}{f^{2} s_{\theta}^{2}} c_{2 \theta}
\end{aligned}
$$

\section{B Potential}

Here we provide some analytical formulas for the potential. The leading order contribution to the potential from gauge loops (neglecting hyper-charge effects) is given by [1],

$$
V(h)_{\text {gauge }} \approx \frac{9}{4} \frac{1}{16 \pi^{2}} \frac{g_{0}^{2}}{g_{\rho}^{2}} \frac{m_{\rho}^{4}\left(m_{a_{1}}^{2}-m_{\rho}^{2}\right)}{m_{a_{1}}^{2}-m_{\rho}^{2}\left(1+g_{0}^{2} / g_{\rho}^{2}\right)} \ln \left[\frac{m_{a_{1}}^{2}}{m_{\rho}^{2}\left(1+g_{0}^{2} / g_{\rho}^{2}\right)}\right] \sin ^{2} \frac{h}{f} .
$$

This formula agrees with our intuition in various ways. In the SM the quadratic divergence of the Higgs mass due to gauge loops reads,

$$
\delta m_{h}^{2}=\frac{9 g^{2}}{32 \pi^{2}} \Lambda^{2}
$$

where $\Lambda$ is a momentum cut-off. Comparing with the formula above we see that the $\mathrm{SO}(4)$ resonances act as the cut-off of the gauge loops. More precisely we see that the quadratic divergence is cut-off at the mass of triplet mixing with SM gauge fields whose mass is $m_{\rho} \sqrt{1+g_{0}^{2} / g_{\rho}^{2}}$. The residual logarithmic sensitivity to the cutoff is regulated by the coset resonances in $\mathrm{SO}(5) / \mathrm{SO}(4)$.

The expressions for the fermions (A.6) are more involved but the physics similar. To be definite let us consider $\mathrm{CHM}_{5}$. To gain some insight into these formulas we can expand around $\Delta_{t_{L}, t_{R}}=0$. To leading order we have,

$$
\begin{aligned}
& \alpha=-\frac{N_{c}}{16 \pi^{2}} \int_{0}^{\infty} d t\left[\frac{\left(m_{1}^{2}-m_{3}^{2}\right)\left(m_{3}^{2}-m_{2}^{2}\right)\left[\Delta_{t_{L}}^{2} m_{Q}^{2} t\left(t+m_{T}^{2}\right)-2 \Delta_{t_{R}}^{2} m_{T}^{2} t\left(t+m_{Q}^{2}\right)\right]}{m_{3}^{2}\left(t+m_{1}^{2}\right)\left(t+m_{2}^{2}\right)\left(t+m_{3}^{2}\right)\left(t+m_{4}^{2}\right)}\right]+\ldots \\
& \beta=\frac{N_{c} \Delta_{t_{L}}^{2} \Delta_{t_{R}}^{2}}{64 \pi^{2}} \int_{0}^{\infty} d t\left[\frac{m_{1} m_{2}\left(m_{1}^{2}-m_{3}^{2}\right)\left(m_{3}^{2}-m_{2}^{2}\right)+m_{3}^{2} Y_{T}^{2}}{Y_{T} m_{3}^{2}\left(t+m_{1}^{2}\right)\left(t+m_{2}^{2}\right)}+\left(\begin{array}{l}
m_{1} \leftrightarrow m_{3} \\
m_{2} \leftrightarrow m_{4}
\end{array}\right)\right]^{2}+\ldots
\end{aligned}
$$

where $t \equiv-p^{2}$ and we have expressed the integrals in terms of the physical masses of the singlets $\left(m_{1,2}\right)$ and quadruplets $\left(m_{3,4}\right)$. in the limit in which we are working each $\mathrm{SO}(4)$ rep is degenerate. Note that the following relations hold in our model,

$$
m_{1} m_{2}=m_{3} m_{4}, \quad y_{t} \approx \frac{\Delta_{t_{L}} \Delta_{t_{R}}}{m_{1} m_{2}} \frac{Y_{T}}{f}
$$

For $\Lambda \ll m_{i}$ the integrals behave as,

$$
\begin{aligned}
\alpha & =\frac{N_{c}}{16 \pi^{2}} \frac{\left(2 \Delta_{t_{R}}^{2}-\Delta_{t_{L}}^{2}\right)\left(m_{1}^{2}-m_{3}^{2}\right)\left(m_{2}^{2}-m_{3}^{2}\right)}{m_{1}^{2} m_{2}^{2} m_{3}^{2}} \Lambda^{4} \\
\beta & =\frac{N_{c} y_{t}^{2}}{16 \pi^{2}} f^{2} \Lambda^{2}
\end{aligned}
$$


From the low energy point of view $\alpha$ is quartically divergent. This contribution originates from the Higgs dependence of the kinetic terms that behaves as the vacuum energy. $\beta$ reproduces the quadratic divergence of the top in the SM with the appropriate coefficient. The divergences are reduced to logarithmic above $m_{1}$ and $m_{3}$. Both the singlet and the quadruplet are necessary to regulate the integrals. This is very similar to supersymmetry where the analogous cancellation is due to left and right stops. The mixing terms in $\beta$ are also reminiscent of the $A$-terms.

The integrals above can be done analytically but the answer is not particularly illuminating. To get a more useful expression we can decouple the heavy singlet and quadruplet by taking $m_{2,4} \rightarrow \infty$ according to eq. (B.4). To get a finite Yukawa we then have to scale $Y_{T}=\kappa \sqrt{m_{2} m_{4}}$. In this limit we can interpret $\kappa \sqrt{m_{1} m_{2}} / f$ as the fermionic coupling of the strong sector. We note that the integral of $\beta$ is finite in this limit while $\alpha$ in general requires also the second layer of resonances to regulate a residual logarithmic divergence. We find,

$$
\begin{aligned}
\beta=\frac{N_{c} y_{t}^{2}}{64 \pi^{2} \kappa^{4}} \times & {\left[\frac{\left(m_{1}^{2}-m_{3}^{2}\right)^{3}\left(m_{1}^{2}+m_{3}^{2}\right)^{2}-2 m_{1} m_{3}\left(m_{1}^{2}-m_{3}^{2}\right)^{3} \kappa^{2}+m_{1}^{2} m_{3}^{2}\left(m_{1}^{4}-m_{3}^{4}\right) \kappa^{4}}{m_{1}^{2} m_{3}^{2}\left(m_{1}^{2}-m_{3}^{2}\right)}\right.} \\
& +\frac{4 m_{1}^{2} m_{3}^{2}\left(m_{1}^{4}+m_{3}^{4}-m_{1}^{2} m_{3}^{2}\left(2+\kappa^{4}\right) \log \frac{m_{3}}{m_{1}}\right] f^{2}}{m_{1}^{2} m_{3}^{2}\left(m_{1}^{2}-m_{3}^{2}\right)}
\end{aligned}
$$

For $m_{1}=m_{3}$ we find simply,

$$
\beta=\frac{N_{c} y_{t}^{2}}{16 \pi^{2}} f^{2} m_{1}^{2} .
$$

$\alpha$ is also calculable for this choice and both contributions (proportional to $\Delta_{t_{L}, t_{R}}^{2}$ ) vanish. Deviating from this point one should be able to find solutions. Indeed in a sizable fraction of our samples the coefficient $\alpha$ is small compared to the naive estimate because it is the sum of small terms rather the cancellation of large terms. Other regions of parameters space however can also be found.

Open Access. This article is distributed under the terms of the Creative Commons Attribution License which permits any use, distribution and reproduction in any medium, provided the original author(s) and source are credited.

\section{References}

[1] S. De Curtis, M. Redi and A. Tesi, The 4d composite Higgs, JHEP 04 (2012) 042 [arXiv: 1110.1613] [INSPIRE].

[2] ATLAS collaboration, G. Aad et al., Combined search for the standard model Higgs boson using up to $4.9 \mathrm{fb}^{-1}$ of pp collision data at $\sqrt{s}=7 \mathrm{TeV}$ with the ATLAS detector at the LHC, Phys. Lett. B $\mathbf{7 1 0}$ (2012) 49 [arXiv: 1202.1408] [INSPIRE].

[3] CMS collaboration, S. Chatrchyan et al., Combined results of searches for the standard model Higgs boson in pp collisions at $\sqrt{s}=7$ TeV, Phys. Lett. B 710 (2012) 26 [arXiv: 1202 .1488] [INSPIRE]. 
[4] D.B. Kaplan and H. Georgi, SU(2) $\times$ U(1) breaking by vacuum misalignment, Phys. Lett. B 136 (1984) 183 [INSPIRE].

[5] H. Georgi, D.B. Kaplan and P. Galison, Calculation of the composite Higgs mass, Phys. Lett. B 143 (1984) 152 [INSPIRE].

[6] H. Georgi and D.B. Kaplan, Composite Higgs and custodial SU(2), Phys. Lett. B 145 (1984) 216 [INSPIRE].

[7] M.J. Dugan, H. Georgi and D.B. Kaplan, Anatomy of a composite Higgs model, Nucl. Phys. B 254 (1985) 299 [INSPIRE].

[8] K. Agashe, R. Contino and A. Pomarol, The minimal composite Higgs model, Nucl. Phys. B 719 (2005) 165 [hep-ph/0412089] [INSPIRE].

[9] B. Gripaios, A. Pomarol, F. Riva and J. Serra, Beyond the minimal composite Higgs model, JHEP 04 (2009) 070 [arXiv: 0902.1483] [INSPIRE].

[10] J. Mrazek, A. Pomarol, R. Rattazzi, M. Redi, J. Serra and A. Wulzer, The other natural two Higgs doublet model, Nucl. Phys. B 853 (2011) 1 [arXiv:1105.5403] [INSPIRE].

[11] G. Panico and A. Wulzer, The discrete composite Higgs model, JHEP 09 (2011) 135 [arXiv:1106.2719] [INSPIRE].

[12] R. Contino, The Higgs as a composite Nambu-Goldstone boson, arXiv:1005.4269 [INSPIRE].

[13] R. Contino, T. Kramer, M. Son and R. Sundrum, Warped/composite phenomenology simplified, JHEP 05 (2007) 074 [hep-ph/0612180] [INSPIRE].

[14] G. Giudice, C. Grojean, A. Pomarol and R. Rattazzi, The strongly-interacting light Higgs, JHEP 06 (2007) 045 [hep-ph/0703164] [INSPIRE].

[15] M. Piai, A. Pierce and J.G. Wacker, Composite vector mesons from QCD to the little Higgs, hep-ph/0405242 [INSPIRE].

[16] H.-C. Cheng, J. Thaler and L.-T. Wang, Little M-theory, JHEP 09 (2006) 003 [hep-ph/0607205] [INSPIRE].

[17] J. Thaler, Little technicolor, JHEP 07 (2005) 024 [hep-ph/0502175] [INSPIRE].

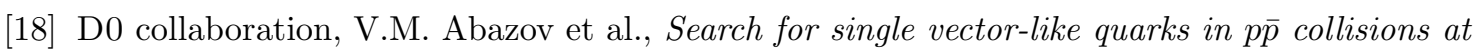
$\sqrt{s}=1.96 \mathrm{TeV}$, Phys. Rev. Lett. 106 (2011) 081801 [arXiv:1010.1466] [INSPIRE].

[19] CMS collaboration, S. Chatrchyan et al., Search for a vector-like quark with charge $2 / 3$ in $t+Z$ events from pp collisions at $\sqrt{s}=7$ TeV, Phys. Rev. Lett. 107 (2011) 271802 [arXiv: 1109.4985] [INSPIRE].

[20] J. Espinosa, C. Grojean, M. Muhlleitner and M. Trott, Fingerprinting Higgs suspects at the LHC, JHEP 05 (2012) 097 [arXiv: 1202 . 3697] [INSPIRE].

[21] D. Carmi, A. Falkowski, E. Kuflik and T. Volansky, Interpreting LHC Higgs results from natural new physics perspective, JHEP 07 (2012) 136 [arXiv:1202.3144] [INSPIRE].

[22] A. Azatov, R. Contino and J. Galloway, Model-independent bounds on a light Higgs, JHEP 04 (2012) 127 [arXiv:1202.3415] [INSPIRE].

[23] P.P. Giardino, K. Kannike, M. Raidal and A. Strumia, Reconstructing Higgs boson properties from the LHC and Tevatron data, JHEP 06 (2012) 117 [arXiv: 1203.4254] [INSPIRE].

[24] J. Ellis and T. You, Global analysis of experimental constraints on a possible Higgs-like particle with mass $\sim 125 \mathrm{GeV}$, JHEP 06 (2012) 140 [arXiv: 1204.0464] [INSPIRE]. 
[25] R. Contino, C. Grojean, M. Moretti, F. Piccinini and R. Rattazzi, Strong double Higgs production at the LHC, JHEP 05 (2010) 089 [arXiv: 1002.1011] [INSPIRE].

[26] M. Redi and A. Weiler, Flavor and CP invariant composite Higgs models, JHEP 11 (2011) 108 [arXiv: 1106.6357] [INSPIRE].

[27] R. Barbieri, D. Buttazzo, F. Sala and D.M. Straub, Flavour physics from an approximate $\mathrm{U}(2)^{3}$ symmetry, JHEP 07 (2012) 181 [arXiv: 1203.4218] [INSPIRE].

[28] M. Redi, Composite MFV and beyond, The European Physical Journal C (2012) 72:2030 [arXiv: 1203.4220] [INSPIRE].

[29] R. Contino, L. Da Rold and A. Pomarol, Light custodians in natural composite Higgs models, Phys. Rev. D 75 (2007) 055014 [hep-ph/0612048] [INSPIRE].

[30] G. Panico, M. Safari and M. Serone, Simple and realistic composite Higgs models in flat extra dimensions, JHEP 02 (2011) 103 [arXiv: 1012.2875] [INSPIRE].

[31] M. Frigerio, A. Pomarol, F. Riva and A. Urbano, Composite scalar dark matter, JHEP 07 (2012) 015 [arXiv: 1204.2808] [INSPIRE].

[32] A. Azatov and J. Galloway, Light custodians and Higgs physics in composite models, Phys. Rev. D 85 (2012) 055013 [arXiv:1110.5646] [InSPIRE].

[33] M. Papucci, J.T. Ruderman and A. Weiler, Natural SUSY endures, JHEP 09 (2012) 035 [arXiv:1110.6926] [INSPIRE].

[34] C. Brust, A. Katz, S. Lawrence and R. Sundrum, SUSY, the third generation and the LHC, JHEP 03 (2012) 103 [arXiv:1110.6670] [INSPIRE].

[35] O. Matsedonskyi, G. Panico and A. Wulzer, Light top partners for a light composite Higgs, arXiv:1204.6333 [INSPIRE]. 